\title{
The antiphase domain structure and parameters of the solid solution in the alloys $\mathrm{Pd}_{3} \mathrm{Fe}$ and $\mathrm{Ni}_{3} \mathrm{Mn}$ with superstructure $L 1_{2}$
}

\author{
E.V. Konovalova ${ }^{1, \dagger}$, O.B. Perevalova ${ }^{2}$, N.A. Koneva ${ }^{3}$, E.V. Kozlov ${ }^{3}$ \\ †knv123@yandex.ru \\ ${ }^{1}$ Surgut State University, Surgut, 628400, Russia, pr. Lenina, 1 \\ ${ }^{2}$ Institute of Strength Physics and Materials Science, SD RAS, Tomsk, 634021 Russia, pr. Academicheskii, 2/4 \\ ${ }^{3}$ Tomsk State University of Architecture and Building, Tomsk, 634003 Russia, pl. Solyanaya, 2
}

By methods of transmission electron diffraction microscopy the antiphase domain structure was investigated and its distributions upon sizes were received in $\mathrm{Pd}_{3} \mathrm{Fe}$ and $\mathrm{Ni}_{3} \mathrm{Mn}$ alloys at the different long-range atomic order degree. The dislocation structure and phase composition of alloys were also investigated. The parameters of the solid solution such as the degree of long-range order and microdistortions were determined by X-ray. The different means of degree of long-range order are achieved by deviations from the stoichiometric composition in $\mathrm{Ni}_{3} \mathrm{Mn}$ alloy and by varying of the annealing regimes in the $\mathrm{Pd} \mathrm{Fe}_{3}$ alloy. The average sizes of antiphase domain increase, their boundaries are become blurring and the manganese oxide particles are allocated on the grain boundaries and antiphase domains with the decreasing of the degree of long-range order in the nonstoichiometric composition $\mathrm{Ni}_{3} \mathrm{Mn}$ alloy. In the $\mathrm{Pd}_{3} \mathrm{Fe}$ the degree of long-range order decreases at the increasing of isothermal annealing duration. It was accompanied by an increasing of the antiphase domain average sizes and blurring their boundaries as in the $\mathrm{Ni}_{3} \mathrm{Mn}$. Besides the degree of long-range order decreasing was accompanied by stacking fault emergencing and average scalar and excess dislocation densities increasing. The observed changes in the alloys microstructure lead to microdistortions increasing. The relationships between degree of long-range order, antiphase domains and microdistortion were established. The antiphase domain average sizes and microdistortions increase at the degree of long-range order decreasing.

Keywords: antiphase domains, degree of long-range order, microdistortion.

\section{Антифазная доменная структура и параметры твердого раствора в сплавах $\mathrm{Pd}_{3} \mathrm{Fe}$ и $\mathrm{Ni}_{3} \mathrm{Mn}$ со сверхструктурой $L 1_{2}$}

\author{
Коновалова Е.В. ${ }^{1 \dagger}$, Перевалова О.Б. ${ }^{2}$, Конева Н.А. ${ }^{3}$, Козлов Э.В. ${ }^{3}$ \\ ${ }^{1}$ Сургутский государственный университет, 628400, г. Сургут, пр. Ленина 1 \\ ${ }^{2}$ Институт физики прочности и материаловедения СО РАН, 634021, г.Томск, пр.Академический, 2/4 \\ ${ }^{3}$ Томский государственный архитектурно-строительный университет, 634003, г.Томск, пл.Соляная, 2
}

Методами просвечивающей электронной дифракционной микроскопии исследована антифазная доменная структура и получены распределения антифазных доменов по размерам в сплавах $\mathrm{Pd}_{3} \mathrm{Fe}$ и $\mathrm{Ni}_{3} \mathrm{Mn}$ со сверхструктурой $L 1_{2}$ при разной степени дальнего атомного порядка, а также исследована дислокационная структура и фазовый состав сплавов. Методами рентгеноструктурного анализа определены параметры твердого раствора такие, как степень дальнего атомного порядка и микродеформация кристаллической решетки. $\mathrm{B}$ сплаве $\mathrm{Ni}_{3} \mathrm{Mn}$ разная степень дальнего атомного порядка достигалась отклонением от стехиометрического состава, в сплаве $\mathrm{Pd}_{3} \mathrm{Fe}-$ путем вариации режима отжига на упорядочение атомов (ступенчатый отжиг и изотермические отжиги при $T<T_{K}$ ). При уменьшении степени дальнего атомного порядка в сплаве $\mathrm{Ni}_{3} \mathrm{Mn}$ нестехиометрического состава происходит увеличение средних размеров антифазных доменов, размытие их границ и выделение частиц оксидов марганца по границам зерен и антифазных доменов. В сплаве $\mathrm{Pd}_{3}$ Fе степень дальнего атомного порядка уменьшается при увеличении длительности изотермических отжигов и сопровождается, как и в сплаве $\mathrm{Ni}_{3} \mathrm{Mn}$, увеличением средних размеров доменов, размытием их границ, а также появлением дефектов упаковки, возрастанием средней скалярной и избыточной плотности дислокаций. Наблюдаемые изменения в микроструктуре исследуемых сплавов приводят к увеличению микродеформации кристаллической решетки. Установлена взаимосвязь между степенью дальнего атомного порядка, размером антифазных доменов и микродеформацией кристаллической решетки. С уменьшением степени дальнего атомного порядка средний размер антифазных доменов и микродеформация кристаллической решетки увеличиваются.

Ключевые слова: антифазные домены, степень дальнего атомного порядка, микродеформация. 
В упорядоченном состоянии сплавов важным дефектом кристаллической структуры являются антифазные границы (АФГ), которые могут быть термического или деформационного происхождения. Термические АФГ возникают при длительных отжигах, проводимых для упорядочения атомов. Отличительной особенностью термических АФГ является их стремление к размытию в процессе отжига [1], то есть к релаксации на большое число атомных плоскостей по обе стороны от границы. Это приводит к тому, что вблизи АФГ параметры твердого раствора (параметр кристаллической решетки, степень дальнего атомного порядка) отличаются от параметров внутри антифазного домена (АФД). Ансамбль АФД влияет на электронную структуру сплава. Экспериментально [1] установлено, что при среднем размере доменов, равном 20 нм и $\delta$-функции распределения доменов по размерам антифазная доменная структура стабилизирует упорядоченную фазу, т.е. влияет на зонную структуру сплава таким образом, что его упорядоченное состояние становится энергетически выгодным.

Цель настоящей работы состоит в установлении взаимосвязи между структурой АФД и параметрами твердого раствора, такими как степень дальнего атомного порядка $\eta$ и микродеформация кристаллической решетки $\varepsilon$.

В качестве материалов исследования выбраны сплавы $\mathrm{Pd}_{3} \mathrm{Fe}$ и $\mathrm{Ni}_{3} \mathrm{Mn}$, упорядочивающиеся через двухфазную область $\left(A 1+L 1_{2}\right)$. Разная степень дальнего атомного порядка в сплавах достигалась различным образом: в $\mathrm{Pd}_{3} \mathrm{Fe}$ путем вариации режима отжига, в $\mathrm{Ni}_{3} \mathrm{Mn}-$ отклонением от стехиометрического состава. В таблице 1 приведены составы исследуемых сплавов и режимы их термообработки на упорядочение атомов.

Методами просвечивающей электронной дифракционной микроскопии исследована структура АФД. Исследования проводили на просвечивающем электронном микроскопе FEI Tecnai 20 G2 TWIN. Степень дальнего атомного порядка определяли методом рентгеноструктурного анализа из соотношения интенсивностей сверхструктурных и основных отражений. Микродеформация кристаллической решетки определялась методом аппроксимации из уширения структурных рентгеновских линий. Исследования проводили на дифрактометре ART X'TRA в излучении $\mathrm{CuK}_{\alpha}$.

Результаты определения степени дальнего атомного порядка в сплавах $\mathrm{Pd}_{3} \mathrm{Fe}$ и $\mathrm{Ni}_{3} \mathrm{Mn}$ после отжигов на упорядочение атомов представлены в таблице 1. Установлено, что в сплаве $\mathrm{Pd}_{3} \mathrm{Fe} \eta$ имеет максимальное значение после ступенчатого отжига. Электронно-микроскопически обнаружено, что доменная структура сплава $\mathrm{Pd}_{3} \mathrm{Fe}$ после ступенчатого отжига является однородной (рис. 1a) и характеризуется наименьшим средним значением размеров АФД по сравнению с доменной структурой образцов после изотермических отжигов (табл. 1). Распределение АФД по размерам в образце после ступенчатого упорядочивающего отжига представлено на рис. 2a.

При увеличении длительности изотермического упорядочивающего отжига до 170 ч доменная структура изменяется (рис. 1с), а именно, становится неоднородной по размерам с размытыми АФГ извилистой формы. Изменяется и функция распределения доменов по размерам (рис. 2b), средний размер АФД увеличивается. Увеличение длительности изотермического отжига до 350 ч приводит к увеличению среднего размера АФД и усилению размытия их границ. Степень дальнего атомного порядка с увеличением среднего размера АФД уменьшается (рис. 3). Увеличение длительности изотермического упорядочивающего отжига также сопровождается появлением дефектов упаковки, увеличением как средней скалярной плотности дислокаций в сплаве, так и избыточной. Последнее приводит к образованию экстинкционных контуров, свидетельствующих о появлении упругой микродеформации кристаллической решетки.

табл. 1. Температура превращения порядок-беспорядок $\left(T_{K}\right)$, температура отжига $\left(T_{\text {om }}\right)$, время отжига, степень дальнего атомного порядка $(\eta)$, средние размеры АФД $(\langle D\rangle)$.

Table 1. Temperature of the order-disorder transition $\left(T_{K}\right)$, annealing temperature $\left(T_{a n n}\right)$, annealing time, degree of long-range order $(\eta)$, APD size $(\langle D\rangle)$.

\begin{tabular}{|c|c|c|c|c|c|}
\hline $\begin{array}{l}\text { сплав } \\
\text { alloy }\end{array}$ & $\begin{array}{c}T_{K} \\
\mathrm{~K}\end{array}$ & $\begin{array}{l}T_{o m}, \mathrm{~K} \\
T_{a n n}, \mathrm{~K}\end{array}$ & $\begin{array}{c}\text { Время } \\
\text { отжига } \\
\text { Annealing } \\
\text { time }\end{array}$ & $\eta \pm 0.02$ & $\begin{array}{l}\langle D\rangle, \mathrm{HM} \\
\langle D\rangle, \mathrm{nm}\end{array}$ \\
\hline \multirow{3}{*}{$\mathrm{Pd}_{3} \mathrm{Fe}$} & \multirow{3}{*}{910} & $\begin{array}{l}\text { Ступенчатый отжиг в интервале } 923 \text { - } 773 \text { К со ско- } \\
\text { ростью } 5 \text { К в сутки, далее охлаждение с печью } \\
\text { Stepped annealing in the interval } 923 \text { - } 773 \mathrm{~K} \text { a rate of } \\
5 \text { K per day, followed by furnace cooling }\end{array}$ & $\begin{array}{l}60 \text { дней } \\
\text { 60days }\end{array}$ & 0.91 & 34 \\
\hline & & $\begin{array}{l}\text { Изотермический отжиг при } 873 \mathrm{~K} \text { Isothermal } \\
\text { annealing at } 873 \mathrm{~K}\end{array}$ & $\begin{array}{l}175 \text { часов } \\
175 \mathrm{~h}\end{array}$ & 0.87 & 80 \\
\hline & & $\begin{array}{l}\text { Изотермический отжиг при } 873 \mathrm{~K} \text { Isothermal } \\
\text { annealing at } 873 \mathrm{~K}\end{array}$ & $\begin{array}{l}350 \text { часов } \\
350 \mathrm{~h}\end{array}$ & 0.70 & 160 \\
\hline $\mathrm{Ni}+25$ at. $\% \mathrm{Mn}$ & \multirow{3}{*}{793} & \multirow{3}{*}{$\begin{array}{l}\text { Ступенчатый отжиг в интервале } 798-573 \text { К со ско- } \\
\text { ростью } 5 \text { К в сутки, далее охлаждение с печью } \\
\text { Stepped annealing in the interval } 798-573 \mathrm{~K} \text { a rate of } 5 \\
\text { K per day, followed by furnace cooling }\end{array}$} & \multirow{3}{*}{$\begin{array}{l}60 \text { дней } \\
60 \text { days }\end{array}$} & 0.89 & 11 \\
\hline $\mathrm{Ni}+30$ at. $\% \mathrm{Mn}$ & & & & 0.79 & 26 \\
\hline $\mathrm{Ni}+27$ at. $\% \mathrm{Mn}$ & & & & 0.76 & 43 \\
\hline
\end{tabular}


Размытие АФГ означает появление градиента параметра кристаллической решетки, что должно приводить к ее искажению, т.е. к появлению упругой микродеформации решетки.

В сплаве $\mathrm{Ni}_{3} \mathrm{Mn}$ степень дальнего атомного порядка является максимальной (0.89) в сплаве с содержанием марганца в сплаве, равным 25 ат. \%. Форма АФД близка к кубоидной с расположением АФГ в плоскостях куба. Средний размер АФД в этом сплаве наименьший среди исследуемых. При уменьшении степени дальнего порядка до 0.79 в сплаве $\mathrm{Ni}+30$ ат. \% Mn АФД приобретают вытянутую форму вдоль направления, близкого к <200>, и залегают также в плоскости куба. По их границам и по границам зерен располагаются нанокристаллические оксиды марганца $\mathrm{Mn}_{3} \mathrm{O}_{4}$. В сплаве состава $\mathrm{Ni}+27$ ат. \% Mn, который характеризуется наибольшим содержанием марганца в твердом растворе (28 ат. \% Mn) и наименьшим значением степени дальнего атомного порядка $(\eta=0.76)$ (табл. 1), в сечении на плоскости фольги АФД имеют форму, как квадрата, так и неправильного четырехугольника с криволинейными стенками (рис. 4). Ширина контраста изображений АФГ достигает 10 нм, что почти соизмеримо с размером АФД. С понижением степени дальнего атомного порядка сплава функция распределения АФД по размерам изменяется, при этом средние размеры АФД увеличиваются (рис. 5). Таким образом, в сплаве $\mathrm{Ni}_{3} \mathrm{Mn}$, как и в сплаве $\mathrm{Pd}_{3} \mathrm{Fe}$, увеличение размеров АФД сопровождается уменьшением степени дальнего атомного порядка (рис. 6).

Помимо размытия АФГ в сплаве $\mathrm{Ni}_{3} \mathrm{Mn}$ нестехиометрических составов происходит выделение оксидных частиц как по границам АФД, так и по границам зерен,
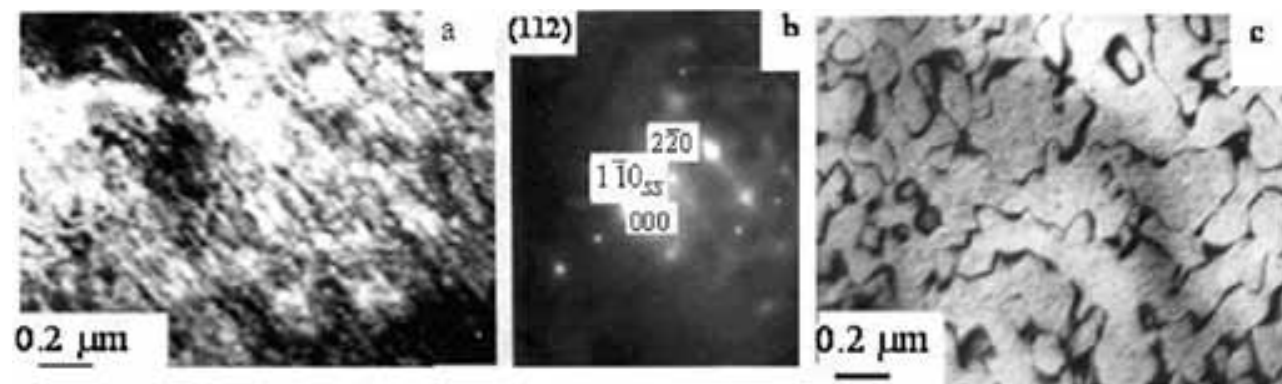

Рис. 1. Доменная структура сплава Pd Fe после ступенчатого упорядочивающего отжига (a, b) и изотермического отжига в течение 170 ч при $873 \mathrm{~K}$ (c): a, c - светлопольные изображения, b - микродифракция.

Fig. 1. Antiphase domain structure in $\mathrm{Pd}_{3} \mathrm{Fe}$ alloy after ordering stepped annealing (a, b) and isothermal annealing at $873 \mathrm{~K}$ for $170 \mathrm{~h}$ : a, $\mathrm{c}-$ bright-field image, $\mathrm{b}$ - microdiffraction.

$$
\delta
$$
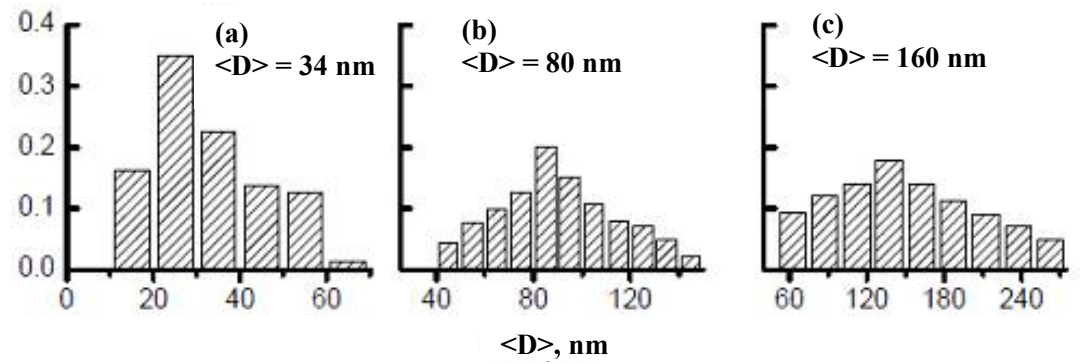

Рис. 2. Распределения АФД по размерам в сплаве $\mathrm{Pd}_{3} \mathrm{Fe}$ (a) - ступенчатый отжиг; (b) - изотермический отжиг при $T=873 \mathrm{~K}$ в течение 175 ч; (c) - изотермический отжиг при $T=873 \mathrm{~K}$ в течение 350 ч.

Fig. 2. Size distribution of antiphase domain structure in $\mathrm{Pd}_{3} \mathrm{Fe}$ alloy: (a) - stepped annealing; (b) - isothermal annealing at $873 \mathrm{~K}$ for 170 $\mathrm{h} ;$ (c) - isothermal annealing at $873 \mathrm{~K}$ for $350 \mathrm{~h}$.

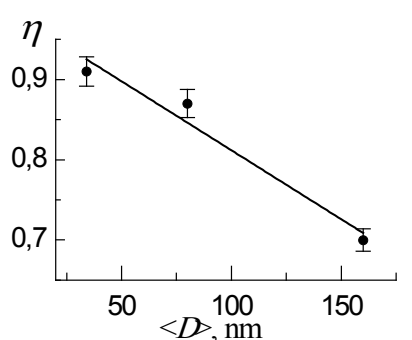

Рис. 3. Зависимость степени дальнего атомного порядка $\eta$ от среднего размера АФД $\langle D\rangle$ в сплаве $\mathrm{Pd}_{3} \mathrm{Fe}$.

Fig. 3. Dependence of the degree of long-range atomic order $\eta$ on the average $\mathrm{APD}\langle D\rangle$ in the $\mathrm{Pd}_{3} \mathrm{Fe}$ alloy
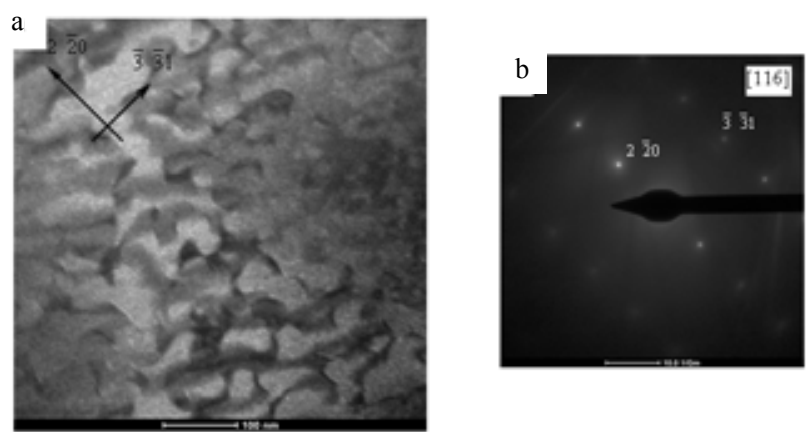

Рис. 4. Антифазная доменная структура в сплаве $\mathrm{Ni}_{3} \mathrm{Mn}$ с содержанием марганца 27 ат. \%: а - темнопольное изображение в сверхструктурном рефлексе (1 $\overline{1} 0) ; \mathrm{b}-$ микродифракция.

Fig. 4. Antiphase domain structure in $\mathrm{Ni}_{3} \mathrm{Mn}$ alloy with the manganece 27 at. \%: a - darkfield image in reflection ( $1 \overline{1} 0)$; (b) - microdiffraction. 
что может приводить к возникновению упругих микронапряжений в кристаллической решетке основной фазы со сверхструктурой $L 1_{2}$.

Методом рентгено-структурного анализа установлено, что полуширина структурных линий в исследуемых сплавах имеет минимальное значение при степени дальнего порядка, близкой к 1 , и увеличивается с уменьшением $\eta$. Поэтому за эталон для каждого сплава было использовано состояние с максимальной степенью дальнего атомного порядка. Из рис. 7 следует, что упругая микродеформация кристаллической решетки увеличивается с уменьшением степени дальнего атомного порядка как в сплаве $\mathrm{Pd}_{3} \mathrm{Fe}$, так и в сплаве $\mathrm{Ni}_{3} \mathrm{Mn}$.

На рис. 8 представлены зависимости среднего размера АФД от микродеформации кристаллической решетки в сплавах $\mathrm{Ni}_{3} \mathrm{Mn}$ и $\mathrm{Pd}_{3} \mathrm{Fe}:$ увеличение микродеформации $\varepsilon$ сопровождается увеличением среднего размера АФД. Указанные зависимости устанавливают всего лишь взаимосвязь между средним размером АФД и микродеформацией решетки. С одной стороны, поскольку увеличение микродеформации решетки увеличивает скорость диффузии [2], то происходит рост АФД. С другой стороны, размытие АФГ при увеличении среднего размера АФД означает уменьшение локальной степени дальнего порядка в объеме, занимаемом АФГ $[1,3]$ и изменение параметра кристаллической решетки в нем по сравнению с телом домена. Последнее приводит к возникновению упругой микродеформации решетки.

Таким образом, увеличение среднего размера АФД в сплавах $\mathrm{Pd}_{3} \mathrm{Fe}$ и $\mathrm{Ni}_{3} \mathrm{Mn}$ со сверхструктурой $L 1_{2}$ происхо- дит при уменьшении степени дальнего атомного порядка и увеличении упругой микродеформации кристаллической решетки.

Благодарность/Aknowledgements. Э.В. Козлов, Н.А. Конева и Е.В. Коновалова проводили исследования в рамках государственного задания Минобрнауки России №3.295.2014/К, О.Б.Перевалова - при финансовой поддержке Программы фундаментальных научных исследований государственных академий наук на 2013-2020 г2.

\section{Литература/References}

1. S. V. Starenchenko, E. V. Kozlov, V. A. Starenchenko. Laws of thermal phase transition of the order-disorder in the alloys with superstructure $L 1_{2}, L 1_{2}(M), L 1_{2}(M M)$, D1a. Tomsk, NTL. (2007) 268 p. (in Russian) [C. B. Старенченко, Э. В. Козлов, В. А. Старенченко. Закономерности термического фазового перехода порядок-беспорядок в сплавах со сверхструктурами $L 1_{2}, L 1_{2}(M), L 1_{2}(M M)$, D1a. Томск, НТЛ. 2007. 268 с.]

2. V. Zait. Diffusion in metals. Moscow, (1958) 381 p. (in Russian) [В. Зайт В. Диффузия в металлах. Москва, Изд-во иностранной литературы. 1958. 381с.]

3. A. A. Chaplygina. Issledovanie structurnyh I sverhstructurnyh prevraschenii v splavah sistemy Cu-Pt. Tomsk. (2013) 23 p. [А. А. Чаплыгина. Исследование структурных и сверхструктурных превращений в сплавах системы Cu-Pt: автореф. канд. физ.-мат. наук. Томск. 2013. 23с.]

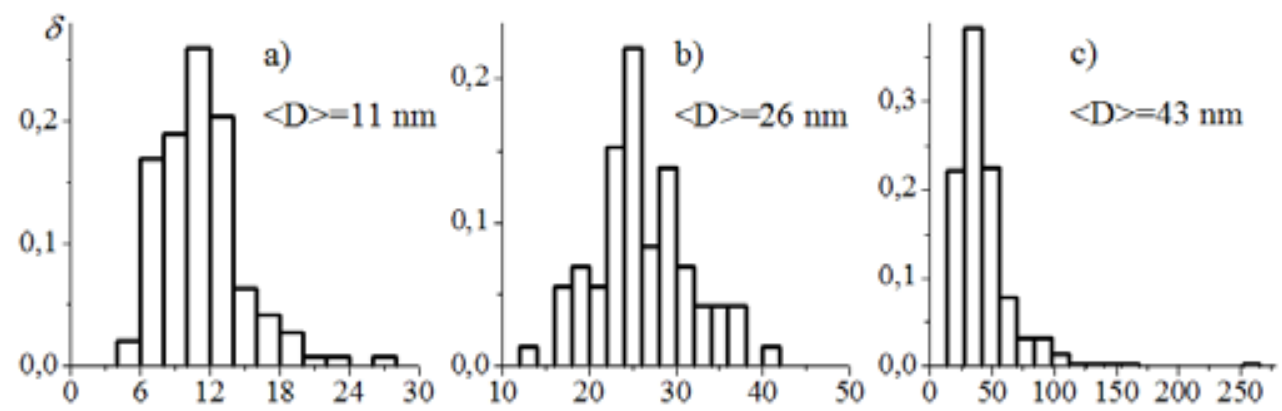

Рис. 5. Распределения по размерам АФД в сплаве $\mathrm{Ni}_{3} \mathrm{Mn}$ с дальним атомным порядком с содержанием марганца (в ат. \%): a) 25; b) 30; с) 27. Fig. 5. Size distribution of antiphase domain structure in $\mathrm{Ni}_{3} \mathrm{Mn}$ ordered alloy with the manganece content (at. \%): a) 25 ; b) 30 ; c) 27 .

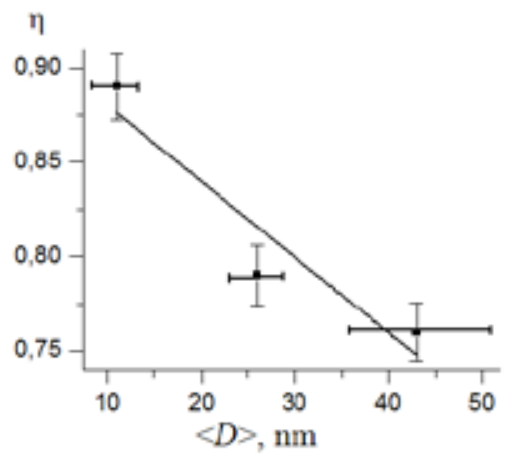

Рис. 6. Зависимость степени дальнего атомного порядка $\eta$ от среднего размера АФД $\langle D\rangle$ в сплаве $\mathrm{Ni}_{3} \mathrm{Mn}$.

Fig. 6. Dependence of the degree of longrange atomic order $\eta$ on the average APD $\langle D\rangle$ in the $\mathrm{Ni}_{3} \mathrm{Mn}$ alloy

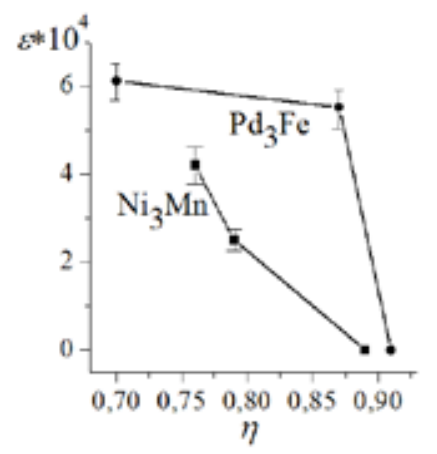

Рис. 7. Зависимость микродеформации кристаллической решетки $\varepsilon$ от степени дальнего атомного порядка $\eta$.

Fig. 7. Dependence of the microdistortions of the crystal lattice $\varepsilon$ on the degree of longrange atomic order $\eta$.

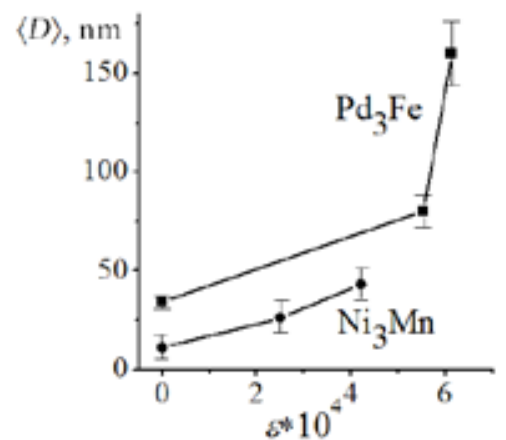

Рис. 8. Зависимость среднего размера АФД $\langle D\rangle$ от микродеформации кристаллической решетки $\varepsilon$.

Fig. 8. Dependence of the average APD $\langle D\rangle$ on the microdistortions of the crystal lattice $\varepsilon$. 